\title{
Integrating rewards and cognition in the frontal cortex
}

\author{
ALISON M. GILBERT and JULIE A. FIEZ \\ University of Pittsburgh, Pittsburgh, Pennsylvania
}

\begin{abstract}
Research indicates that the dorsolateral prefrontal cortex (DLPFC) contributes to working memory and executive control, whereas the ventral frontal cortex (VFC) contributes to affective and motivational processing. Few studies have examined both the functional specificity and the integration of these regions. We did so using fMRI and a verbal working memory task in which visual cues indicated whether recall performance on an upcoming trial would be linked to a monetary reward. On the basis of prior findings obtained in delayed response tasks performed by nonhuman primates, we hypothesized that (1) VFC would show an increase only in response to a cue indicating potential for a monetary reward; (2) DLPFC would show sustained activity across a delay interval for all trials, though activity in rewarded trials would be enhanced; and (3) regions engaged in speech-based rehearsal would be relatively insensitive to monetary incentive. Our hypotheses about DLPFC and rehearsal-related regions were confirmed. In VFC regions, we failed to observe statistically significant effects of reward when the cue or delay epochs of the task were examined in isolation. However, an unexpected and significant deactivation was observed in VFC during the delay epoch; furthermore, a post hoc voxelwise analysis indicated a complex interaction between (1) the cue and delay epochs of the task and (2) the reward value of the trials. The pattern of activation and deactivation across trial types suggests that VFC is sensitive to reward cues, and that portions of DLPFC and VFC may work in opposition during the delay epoch of a working memory task in order to facilitate task performance.
\end{abstract}

Over the past few decades various techniques have been used to identify and characterize brain regions that represent and respond to affective and rewarding stimuli (for a review, see Rolls, 1999). A limitation of research on brain regions associated with reward processing, however, is that it fails to explain how information about potential rewards is integrated via other brain systems to influence higher level cognitive processes and goaldirected behavior. One suggestion is that motivation and cognition are integrated via connections between ventral frontal brain regions involved in the processing of rewardrelated stimuli and dorsolateral prefrontal brain regions associated with cognitive processes (Haber, Fudge, \& McFarland, 2000; Hikosaka \& Watanabe, 2000; Schultz, Tremblay, \& Hollerman, 2000).

In order to establish the specificity and integration of frontal regions involved in motivation and cognition, we employ a verbal delayed recall task in which reward-related cues are used to enhance cognitive task performance. Each trial consists of a reward cue epoch indicating the potential to earn a reward through task performance, followed by an encoding epoch in which a set of items (two-

The authors acknowledge Stephen J. Wilson, Jeff Phillips, Nicole Hill, Kate Fissell, and Jason Chein for their technical assistance, Elizabeth Tricomi for her feedback, and Corrine Durisko for her invaluable support. This work was supported by NIH Grant RO1 DA14103. Correspondence should be addressed to A. M. Gilbert, 619 LRDC, 3939 O'Hara Street, University of Pittsburgh, Pittsburgh, PA 15260 (e-mail: alm17@pitt.edu). syllable words, four-syllable words, or nonwords) is presented and remembered over a delay epoch. The trial ends with a recall epoch during which (as indicated during the cue epoch) a monetary reward is earned on the basis of the participant's ability to correctly recall the remembered items.

The task was designed to be conceptually similar to delayed response tasks performed by nonhuman primates (Hikosaka \& Watanabe, 2000; Leon \& Shadlen, 1999; Schultz et al., 2000), the results of which provide a basis for generating a set of experimental hypotheses. One of these hypotheses is that the ventral frontal cortex (VFC; broadly defined to include Brodmann areas 10, 11, 12, and 47 in the human) is centrally involved in processing information about rewards and thus will respond differentially on reward versus no-reward trials of the delayed serial recall task. This first hypothesis is consistent with single-unit recording data which indicate that neurons in VFC fire in response to a reward cue, exhibit sustained firing in expectation of a reward, and additionally fire in response to the delivery of a reward (Hikosaka \& Watanabe, 2000; Schultz et al., 2000; Tremblay \& Schultz, 2000).

Three specific accounts of the function of sustained delay activity in VFC have been suggested. To avoid confounding the reward cue and delay epochs, Tremblay and Schultz (2000) imposed two delays in a rewarded movement/ nonmovement task. The first delay was imposed between presentation of the reward cue and trigger, and the second delay was imposed following a movement trigger and prior to the receiving of a reward. Fifty-four percent of neurons 
responded to the reward cue, but only $.02 \%$ of neurons exhibited significant sustained activation throughout the first delay. During the second delay, $27 \%$ of neurons in VFC exhibited significant sustained delay-related activation. The larger percentage of neurons exhibiting sustained delay activity during the second delay suggested that delay-related activity is primarily associated with the expectation of reward, although another interpretation was that the number of VFC neurons that exhibit sustained delay activity is modulated by memonic and behavioral task demands.

Hikosaka and Watanabe (2000) found that a subset of neurons in VFC exhibited sustained firing following a cue that indicated future presentation of one of several stimuli that varied in perceived reward value (i.e., potato, raisin, cabbage, no reward). The reward cue was followed by a single delay period and subsequent motor response. Some neurons discriminated among rewards that differed in their incentive value, which suggested that delay-related activity may also encode the motivational value of an anticipated reward. Finally, Schoenbaum and Setlow (2001) have proposed that sustained activation of VFC neurons may also serve to hold information about rewards in working memory (i.e., the delay activity also subserves a mnemonic function). Support for this claim comes from a study in which rats with and without lesions to the orbitofrontal cortex were assessed on a delayednonmatching-to-sample task involving different odors (Otto \& Eichenbaum, 1992). Rats with lesions to VFC required more time to learn the rules of the task than did sham-lesioned animals, and their performance across the delay intervals was significantly worse. Although the tree preceding accounts do not permit one to come to any single conclusion, they do provide several interesting predictions about the pattern of activation that may be observed in VFC. If delay-related activity is primarily associated with expectation of reward, a positive activation should emerge during the recall epoch of our task, prior to the end of each trial. If delay-related activity encodes the emotional value of the anticipated reward or if it functions mnemonically, there should be sustained positive activity during the delay epoch.

A second experimental hypothesis based on the results of nonhuman primate delayed response findings is that the dorsolateral prefrontal cortex (DLPFC, defined as BA 9 and BA 46) is involved in cognitive processing (i.e., executive control) and thus should exhibit delayrelated activity that may be influenced by reward-related processing. However, in contrast to VFC, the DLPFC should show effects of reward only after a memory task has been initiated. In general, early studies that investigated the functional significance of DLPFC used rewards to motivate animals to perform delay tasks, but not in order to determine how rewards influence neuronal activity (e.g., see Funahashi, Bruce \& Goldman-Rakic, 1989).

In several recent studies, however, researchers have manipulated rewards in the context of a delayed response task (Hikosaka \& Watanabe, 2000; Kobayashi, Lauw- ereyns, Koizumi, Sakagami, \& Hikosaka, 2002; Leon \& Shadlen, 1999; Watanabe, 1996; Watanabe, Hikosaka, Sakagami, \& Shirakawa, 2002). For instance, in a study by Leon and Shadlen (1999), monkeys fixated a central point during which a peripheral spatial/memory cue appeared briefly. After a delay, the fixation point disappeared, indicating that the monkey should make a saccade to the remembered target location. A reward cue (the fixation point, in green or red) suggested the amount of reward (small or large) that would be delivered for eye movements to the correct position. On half of the trials, this reward cue appeared during the memory period and after the spatial/memory cue. On the other half of the trials, the reward cue did not occur during the memory period, but, rather, occurred prior to the spatial/memory cue. The responses of neurons in DLPFC were enhanced by the reward only after the stimulus to be remembered was presented. This novel finding suggests that the DLPFC must be recruited by a cognitive task in order to be influenced by affective stimuli.

Leon and Shadlen's (1999) findings are also supported by a human study conducted by Perlstein, Elbert, and Stenger (2002). In this study, participants were randomly presented with pleasant, unpleasant, and neutral singlecue images, followed by a delay interval. Then a probe matrix consisting of nine images was presented randomly on $30 \%$ of the trials. The participants were asked to identify the previously presented image within the matrix. Performance on this working memory task was compared with performance during a similar task (detection task) that varied affective valence, but did not have a memory component since it did not require that participants remember a cued image. Affective valence of the stimulus items was found to modulate activation in the dorsolateral prefrontal cortex only in the context of the working memory task.

A third hypothesis is that frontal brain regions associated with specific rehearsal processes will be relatively unaffected by motivational context. Partial support for this hypothesis comes from considering the role of DLPFC and the frontal eye fields in delayed saccade tasks. Neurons in both the DLPFC and frontal eye fields are activated during the delay period of spatial working memory tasks, and are therefore thought to be involved in visuospatial memory (Funahashi et al., 1989; Hikosaka \& Watanabe, 2000). However, unlike the dorsolateral prefrontal cortex, the frontal eye fields may not be modulated by reward cues during a memory-guided saccade task (Leon \& Shadlen, 1999; for a contradictory result, see Roesch \& Olson, 2003).

Leon and Shadlen's (1999) findings can be explained by Baddeley's (1986) model of working memory. This model includes a domain-general "central executive" responsible for the manipulation of information, and domainspecific components used for the rehearsal of verbal and spatial information. Potentially, the frontal eye fields may specifically contribute to visuospatial rehearsal, and this type of processing may be relatively unaffected by motivational state. We will test this idea in the verbal do- 
main by comparing the effects of reward cues on DLPFC with the effects of reward cues on three areas associated with engagement of a verbal rehearsal strategy: two regions located within the left inferior frontal gyrus (a dorsal region located at or near BA 44/6, and a ventral region located at or near BA 45 and the anterior insula), and a medial region located at or near the supplementary motor area (SMA). In a previous study, we found that activity in these rehearsal-related regions was modulated by factors thought to influence subvocal articulation (e.g., lexical status and syllabic length of stimuli). In contrast, whereas the DLPFC was robustly engaged by the working memory task, its level of activity was only weakly and insignificantly modulated by the stimulus manipulations of length and lexicality (Chein \& Fiez, 2001). In the present study, we sought to demonstrate that the converse pattern holds for the manipulation of reward-specifically, that dorsolateral prefrontal cortex will be engaged by a verbal working memory task, and that the level of activity will be modulated by a reward cue, but not by lexicality and syllabic length of the stimulus items. Conversely, although rehearsal-related regions will also be engaged by a verbal working memory task, the level of activity will not be modulated by a reward cue, but will be modulated by the lexicality and syllabic length of the stimulus items.

\section{METHOD}

\section{Participants}

Twenty-two right-handed native English-speaking undergraduate students ages 18-23 from the University of Pittsburgh and surrounding area were recruited through posted advertisements. They gave their informed consent, in accordance with the policies of the Institutional Review Board at the University of Pittsburgh, prior to their participation. The participants were paid from $\$ 50$ to $\$ 67$ for their participation. Payment was based on performance in the study.

\section{Design and Procedure}

The participants were asked to perform 36 trials of a delayed serial recall task, using a $3 \times 2$ design with list type (two-syllable words, four-syllable words, and nonwords) and reward condition (reward, no reward) as factors. Prior to the testing session, the participants read aloud a list of the stimulus items in order to familiarize themselves with the test materials. The participants were also given one rewarded trial as practice, in which a set of five onesyllable words (cat, send, time, lock, go) was presented.

The participants performed a delayed serial recall task with three types of stimulus lists: two-syllable word lists, four-syllable word lists, and nonword lists. Each trial began with presentation of five two-syllable words, five four-syllable words, or five nonwords. Each list of five items was drawn randomly without replacement, and the five items in each list were randomly ordered. There were 12 trials for each of the three list types, pseudorandomly ordered within each testing session. The different word lists were matched for frequency of occurrence (Francis \& Kučera, 1982).

The participants performed the delayed serial recall task under two reward conditions: $\$ 0$ and $\$ 0.25$. Before each trial the participants were shown the reward amount $(\$ 0$ or $\$ 0.25$ ) for a period of $5 \mathrm{sec}$ and were instructed that they would receive this reward for each word that they recalled in the correct serial position. Half of the lists were associated with no reward, and half were associated with $\$ 0.25$ reward for each correctly recalled item. In addition to a reward cue displayed on the screen prior to the delayed serial recall task, reward amounts were further associated with each trial by altering the color of the background display. Throughout the nonrewarded trials, the background display was colored red; throughout the rewarded trials, the background display was colored green. Thus, the delayed serial recall task consisted of six trials for each condition ( $\$ 0$, two-syllable words $/ \$ 0.25$, two-syllable words $/ \$ 0$, four-syllable words $/ \$ 0.25$, four-syllable words $/ \$ 0$, nonwords $/ \$ 0.25$, nonwords), for a total of 36 trials (Figure 1).

Each $63-\mathrm{sec}$ trial of the delayed serial recall task consisted of five epochs: (1) a reward cue epoch, in which $\$ 0$ or $\$ 0.25$ was displayed (6 sec); (2) an encoding epoch (15 sec), in which five stimuli were sequentially displayed; (3) a delay (maintenance) epoch (18 sec), in which the participants silently rehearsed the list of five stimuli that were presented; (4) a retrieval epoch $(6 \mathrm{sec})$, in which the participant recalled aloud the list of five stimuli; and (5) a fixation epoch $(18 \mathrm{sec})$, in which the participant simply maintained fixation on a rehearsal crosshair. The participants' responses were scored on line, and at the end of the scan session, participants were informed of their total earnings.

Scanning was conducted in a $1.5 \mathrm{GE}$ Signa whole-body magnet with a standard radio frequency coil. To reduce the likelihood of head movement during the scanning session, the participants were reminded to remain still prior to scanning and between each block. Also, during scanning, padding was placed under the participant's head in order to minimize head movement. The task stimuli were projected onto a visual display located behind the participant's head; they were viewed though a mirror placed above and in front of the participant's head. An MRI compatible microphone was used to hear the participants' responses during the scanning session and to transmit verbal responses to a computer program, Cool Edit 2000 (Johnston, 2000), used to digitally record the spoken output. After scanning, the digital recordings were analyzed to confirm the online scoring of participant responses.

Thirty-eight structural contiguous slices $(3.75 \times 3.75 \times 3.8 \mathrm{~mm}$ voxels) parallel to the AC-PC line (at the middle, 20th slice) were acquired using a standard $\mathrm{T} 1$ weighted spin echo pulse sequence. Thirtyeight functional images were acquired at the same oblique axial plane and with the same coverage as that for the structural slices, thus giving us full brain coverage. These images were acquired using a T2* weighted, one-shot reverse spiral pulse sequence $[\mathrm{TR}=3000$, TE $=$ $35 \mathrm{msec} ; \mathrm{FOV}=24$; flip angle $=70^{\circ}$, slice thickness $=3.8 \mathrm{skip}$ $0 \mathrm{~mm}$ ] that enabled a full set of images to be acquired every $3 \mathrm{sec}$.

\section{Analyses}

fMRI analyses were conducted off line, using the NeuroImaging Software package (NIS Version 3.5) developed at the University of Pittsburgh and Princeton University. Automated Image Registration (AIR, Version 3.08) (Woods, Mazziotta, \& Cherry, 1993) was used to reconstruct images and to correct for motion. Images in which motion exceeded $3 \mathrm{~mm}$ or $3^{\circ}$ in any direction were not included in the analysis. In order to adjust for scanner drift between runs, images were linearly detrended. Structural images of each subject were co-registered to a common reference brain (Woods et al., 1993). Functional images were transformed into the same common space, normalized to minimize mean differences in image intensity across subjects, and smoothed with a 3-D Gaussian filter ( $4 \mathrm{~mm}$ FWHM) to account for anatomic variation between subjects. Statistical analyses were then conducted, and time-series data were examined using NIS 3.5. Statistical maps were transformed into Talairach stereotaxic coordinate space using AFNI 2.55j (Cox, 1996).

The analysis focused on identifying and then characterizing the activation patterns in a priori regions of interest. To begin, we used a voxelwise contrast to identify clusters of significant change in our delayed serial recall task, collapsed across the reward and stimulus list conditions. We conducted two such omnibus contrasts. To identify cue-related changes, images acquired 3-9 sec (Scans 2 and 3) after the onset of the reward cue were contrasted to images acquired during the last $6 \mathrm{sec}$ of activity during the fixation epoch (Scans 20 and 21), 


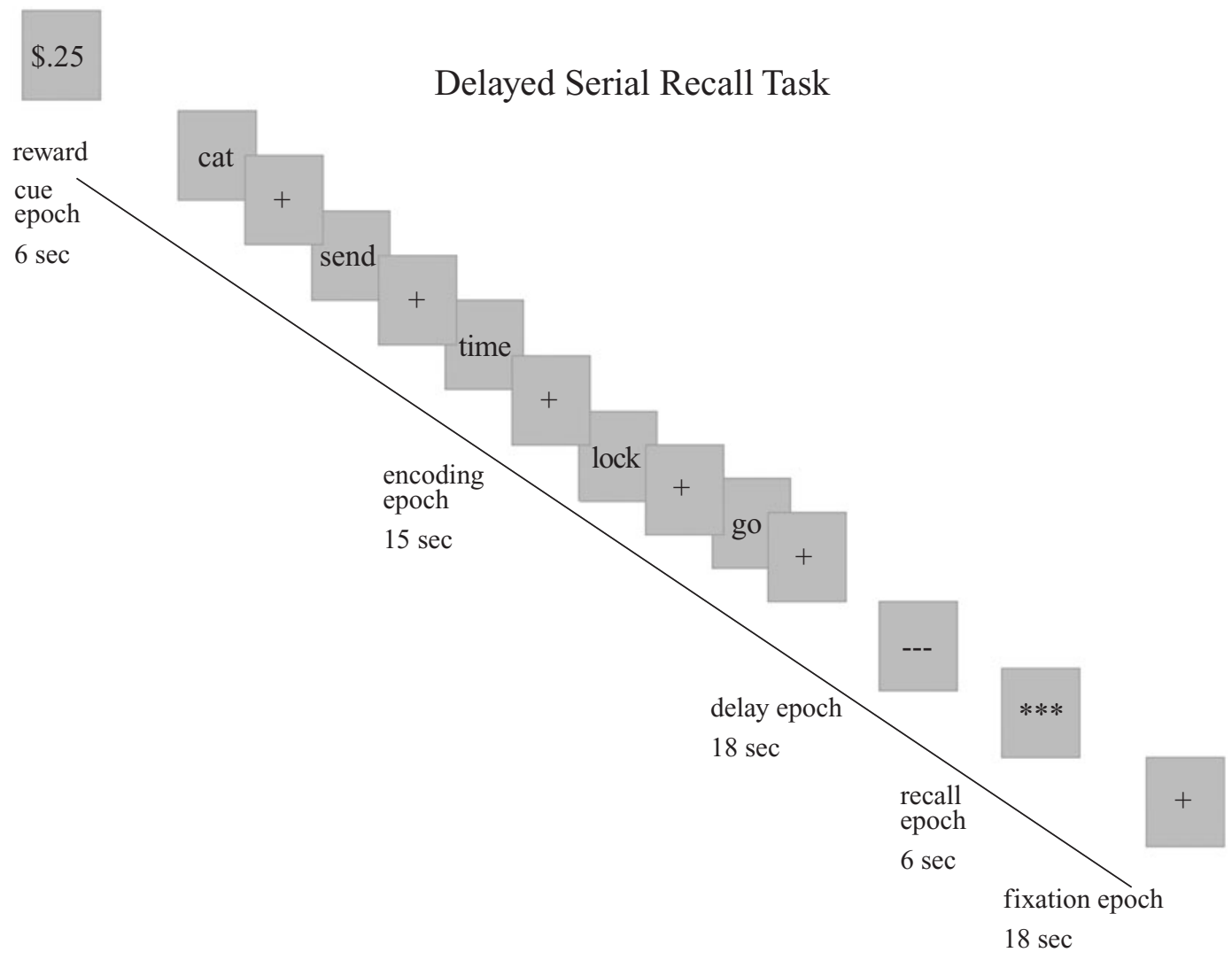

Figure 1. Schematic of the five epochs associated with each trial in the delayed serial recall task: (1) a reward epoch, in which a cue indicates whether a monetary reward will be earned for each successfully recalled stimulus; (2) an encoding epoch, in which a list of five items is serially presented; (3) a delay epoch, during which the stimulus items must be maintained in memory; (4) a recall epoch, in which the previously presented items are recalled aloud; and (5) a fixation epoch, in which subjects maintain visual fixation on a crosshair.

through a series of $t$ tests computed on a voxelwise basis. To identify delay-related changes, images acquired in the first $6 \mathrm{sec}$ of the delay epoch (Scans 8 and 9) were contrasted to images acquired during the last $6 \mathrm{sec}$ of activity during the fixation epoch (Scans 20 and 21), through a series of $t$ tests computed on a voxelwise basis. Prior work indicated that this contrast should capture activity related to stimulus encoding and rehearsal (Chein \& Fiez, 2001).

For both omnibus analyses, clusters of three or more contiguous voxels that exceeded a $t$ value of 2.52 ( $p<.001$ corrected for cluster size, degrees of freedom, and number of sampled voxels) (AFNI AlphaSim; Ward, 2000) were considered to be significantly engaged by the task. We searched within the set of these significant clusters to identify those that fell within a set of a priori regions of interest. As discussed in the introduction, these regions were derived from the neurophysiological literature in which manipulations of rewards have been employed in the context of delayed response tasks. Putative rehearsal-related clusters of activation were those that fell within the left dorsal and ventral inferior frontal cortex (BA 44/6 and the opercular region near the border between BA 45 and the anterior insula), and the supplementary/presupplementary motor area (medial BA 6). Putative regions for executive control were the left and right DLPFC (BA 9/46). Putative clusters involved in the representation of rewards were those located within the VFC (BA 10, 11, 12, and 47).

For each cluster that fell within an a priori region of interest, activity during the cue and delay epochs was probed in more detail.
Specifically, for each cluster, an average time course of activation for each type of trial in each subject was computed. These image intensity values were then assessed with 2 (reward/no-reward) $\times 3$ (two-syllable, four-syllable, nonwords) repeated measures analyses of variance (ANOVAs). One ANOVA examined reward and list effects in the cue epoch (Scans 2 and 3, assuming a 3-sec hemodynamic lag). A second ANOVA examined reward and list effects during the encoding and delay interval (Scans 6-13).

\section{RESULTS}

\section{Behavioral Results}

Behavioral data from the 22 participants were analyzed using a 2 (reward/no reward) $\times 3$ (two-syllable words, four-syllable words, nonwords) ANOVA. Participants performed more accurately on rewarded trials $(75 \%)$ than on nonrewarded trials $(67 \%)[F(1,21)=$ $15.53, p<.001]$. Effects of list type were also statistically significant $[F(2,21)=62.55, p<.0001]$. Participants performed most accurately when asked to recall two-syllable words $(83 \%)$, less accurately when asked to recall nonwords $(71 \%)$, and least accurately when asked to recall four-syllable words (59\%) (Figure 2). 


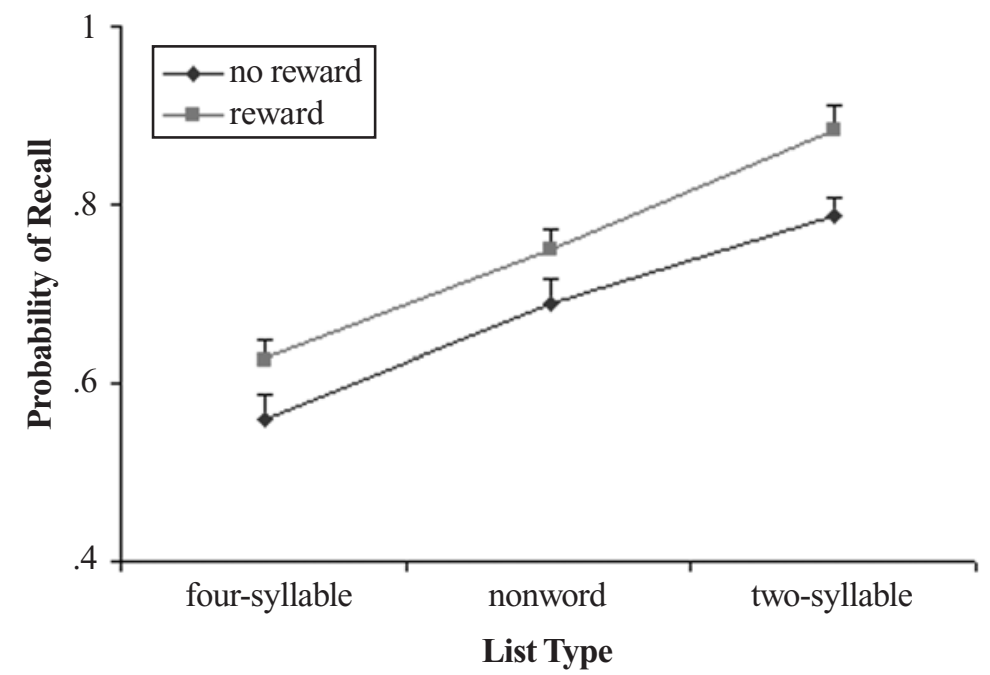

Figure 2. Behavioral effects of reward condition and list type: Probability of successful recall is shown on the $y$-axis for three different types of stimulus items (four-syllable [long] words, nonwords, and two-syllable [short] words). Performance is better for trials associated with a monetary incentive (filled squares) than for those associated with no incentive (filled diamonds).

\section{Imaging Results}

We used contrasts between cue-related activity and the fixation baseline, and between encoding/delay-related activity and the fixation baseline to identify regions of interest for further investigation. In our cue-related contrast, no voxel clusters within our a priori regions of interest survived our significance threshold (regions in VFC were identified at a less stringent threshold). In our delay-related contrast, six voxel clusters fell within our a priori anatomical regions of interest. Three clusters were in putative rehearsal-related regions: the supplementary motor area (SMA), and within the left inferior frontal cortex dorsally at or near BA 44/6, and ventrally at or near BA 45. We also identified a cluster in a putative executive control region, the right DLPFC (BA 9/46). Finally, two putative reward-related clusters fell within the VFC (at or near BA 47, in both the left and the right hemispheres). For each voxel cluster, we conducted repeated measures ANOVAs designed to detect effects of reward $(\$ 0, \$ 0.25)$ and list type (two-syllable words, four-syllable words, and nonwords) during both the cue and encoding/delay epochs. The results, described below, are summarized in Table 1.

Rehearsal-related processing. Turning first to our rehearsal-related clusters in the left inferior frontal cortex (BA 44/6 and BA 45) and supplementary motor area

Table 1

Effects of Reward and List Type in Regions of Interest Identified During Maintenance (Scans 8,9) Versus Fixation (Scans 20,21) $(p<.001$, Corrected)

\begin{tabular}{|c|c|c|c|c|c|c|c|c|c|c|}
\hline \multirow[b]{2}{*}{ Region } & \multirow[b]{2}{*}{ BA } & \multicolumn{3}{|c|}{ Stereotaxic Coordinates } & \multicolumn{2}{|c|}{ Reward } & \multicolumn{2}{|c|}{ List } & \multicolumn{2}{|c|}{ Reward $\times$ List } \\
\hline & & $x$ & $y$ & $z$ & Cue & Delay & Cue & Delay & Cue & Delay \\
\hline \multicolumn{11}{|l|}{ Rehearsal-related regions } \\
\hline Left dorsal inferior frontal cortex & $44 / 6$ & -35 & -13 & 31 & & & & $\begin{array}{l}\mathrm{Nw}>\mathrm{Sh} \\
\mathrm{Lg}>\mathrm{Sh}\end{array}$ & & \\
\hline Left ventral inferior frontal cortex & 45 & -28 & 24 & 8 & & & & $\mathrm{Lg}>\mathrm{Sh}$ & & \\
\hline Supplementary motor cortex & 6 & -2 & 8 & 51 & & & & $\begin{array}{l}\mathrm{Lg}>\mathrm{Sh} \\
\mathrm{Nw}>\mathrm{Sh}\end{array}$ & & \\
\hline \multicolumn{11}{|l|}{ Reward-related regions } \\
\hline Right ventrolateral cortex & 47 & 43 & 30 & -6 & & & $\begin{array}{l}\mathrm{Nw}>\mathrm{Lg} \\
\mathrm{Sh}>\mathrm{Lg}\end{array}$ & $\mathrm{Sh}>\mathrm{Lg}$ & & \\
\hline Left ventrolateral cortex & 47 & -43 & 34 & -6 & & & $\mathrm{Sh}>\mathrm{Lg}$ & $\begin{array}{l}\mathrm{Nw}>\mathrm{Lg} \\
\mathrm{Sh}>\mathrm{Lg}\end{array}$ & & \\
\hline \multicolumn{11}{|l|}{ Executive control } \\
\hline Right dorsolateral prefrontal cortex & $9 / 46$ & 32 & 32 & 32 & & $\mathrm{R}>\mathrm{NR}$ & & $\begin{array}{l}\mathrm{Lg}>\mathrm{Nw} \\
\mathrm{Lg}>\mathrm{Sh}\end{array}$ & & \\
\hline
\end{tabular}

Note-Sh, two-syllable words; Lg, four-syllable words; Nw, nonwords; R, reward condition; NR, nonreward condition. List, reward, and reward $\times$ list effects are $p<.05$. Effects are reported as $x>y$, meaning that mean intensity is greater for condition $x$ than for condition $y$. 

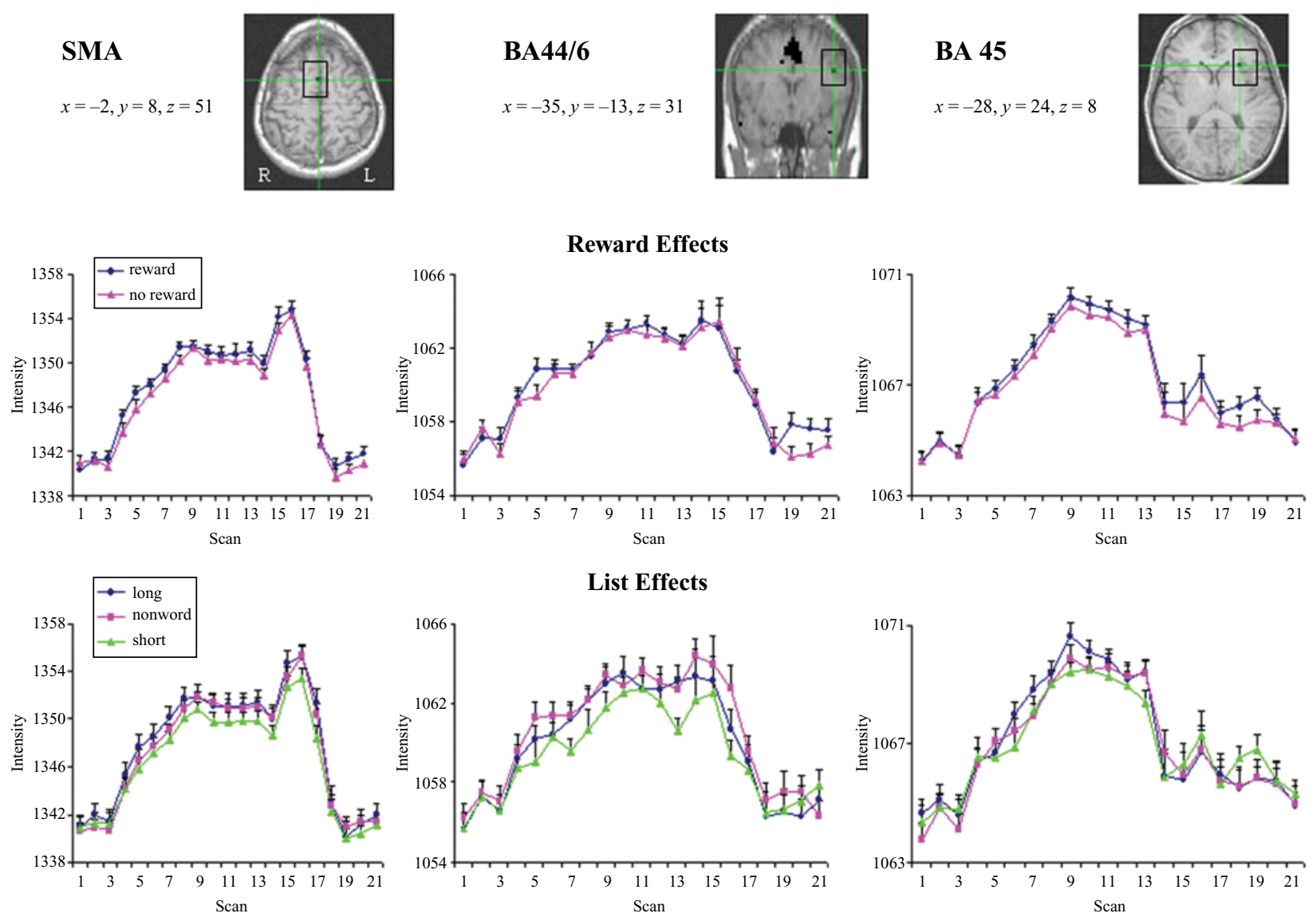

List Effects
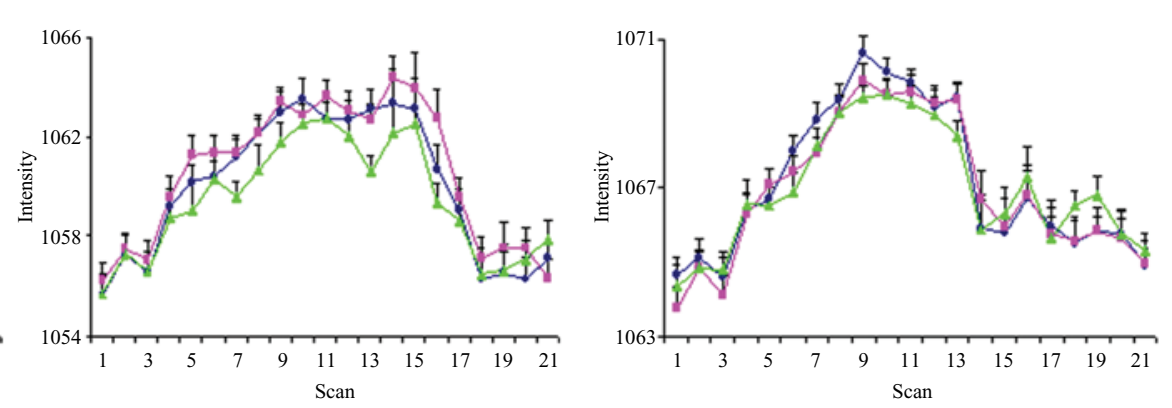

Figure 3. Reward and list type effects in regions associated with verbal rehearsal: Voxel clusters in the supplementary motor area (BA 6, left), dorsal sector of the inferior frontal gyrus (BA 44/6, middle), and ventral sector of the inferior frontal gyrus (BA 45, right) showed an increase in activity during the delay in comparison with the fixation baseline epochs. As is graphically illustrated through the average time course of activity across a trial (21 scans; $63 \mathrm{sec}$ ), all three clusters showed no significant effect of reward cue type (top graph for each cluster), but significantly greater activation for the more difficult stimulus list conditions (bottom graph for each cluster). Our analyses focused on two epochs of interest: the cue epoch (Scans 2 and 3; 3-9 sec), and the delay epoch (Scans 6-13; 15-39 sec).

(BA 6), as expected, during the cue epoch we found no significant effects of reward or list type $(p>.30$ for all effects) (Figure 3). As also predicted, during the delay epoch we did not find significant effects of reward $(p>$ $.05)$. Replicating prior findings, we found that all three rehearsal-related regions were highly sensitive to list type $(p<.05)$, though there were some subtle differences in the ordering of conditions relative to those in a previous study (Chein \& Fiez, 2001). These differences likely reflect the use of two-syllable and four-syllable words in the present study, as compared with the twosyllable and one-syllable words used previously. Finally, there were no significant interactions between reward and list type in any region, during either the cue or the delay epoch ( $p>.35$ for all effects).

Executive processing. In the right dorsolateral prefrontal cortex, a region often associated with executive control (Cohen et al., 1997; Sharp, Scott, \& Wise, 2004), we confirmed that neither reward condition $[F(1,21)=$ $2.12, p=.16]$ nor list type $[F(2,21)=0.094, p=.91]$ in- fluenced brain activation during the cue period of the delayed serial recall task (Figure 4).

As predicted, during the delay epoch, we observed greater activation for reward than for no-reward trials $[F(1,21)=10.97, p=.003]$. On the basis of prior results (Chein \& Fiez, 2001), we anticipated a nonsignificant effect of list type in the right DLPFC. Although our effect size was relatively small, in this larger group of subjects with more demanding stimulus conditions, we observed a highly significant effect of stimulus type $[F(2,21)=$ $7.77, p<.001]$. Finally, as predicted, there were no interactions between reward and list type during either the cue $[F(2,21)=0.05, p=.95]$ or the delay $[F(2,21)=$ $0.63, p=.54]$ epochs.

Reward-related processing. We identified voxel clusters bilaterally in VFC (BA 47) (Figure 5). We predicted that reward versus no-reward trials would produce greater activation during the cue epoch, and potentially during the delay epochs as well. In the cue epoch, the effects of reward were not significant in either the right 
BA9/46

$x=32, y=32, z=32$
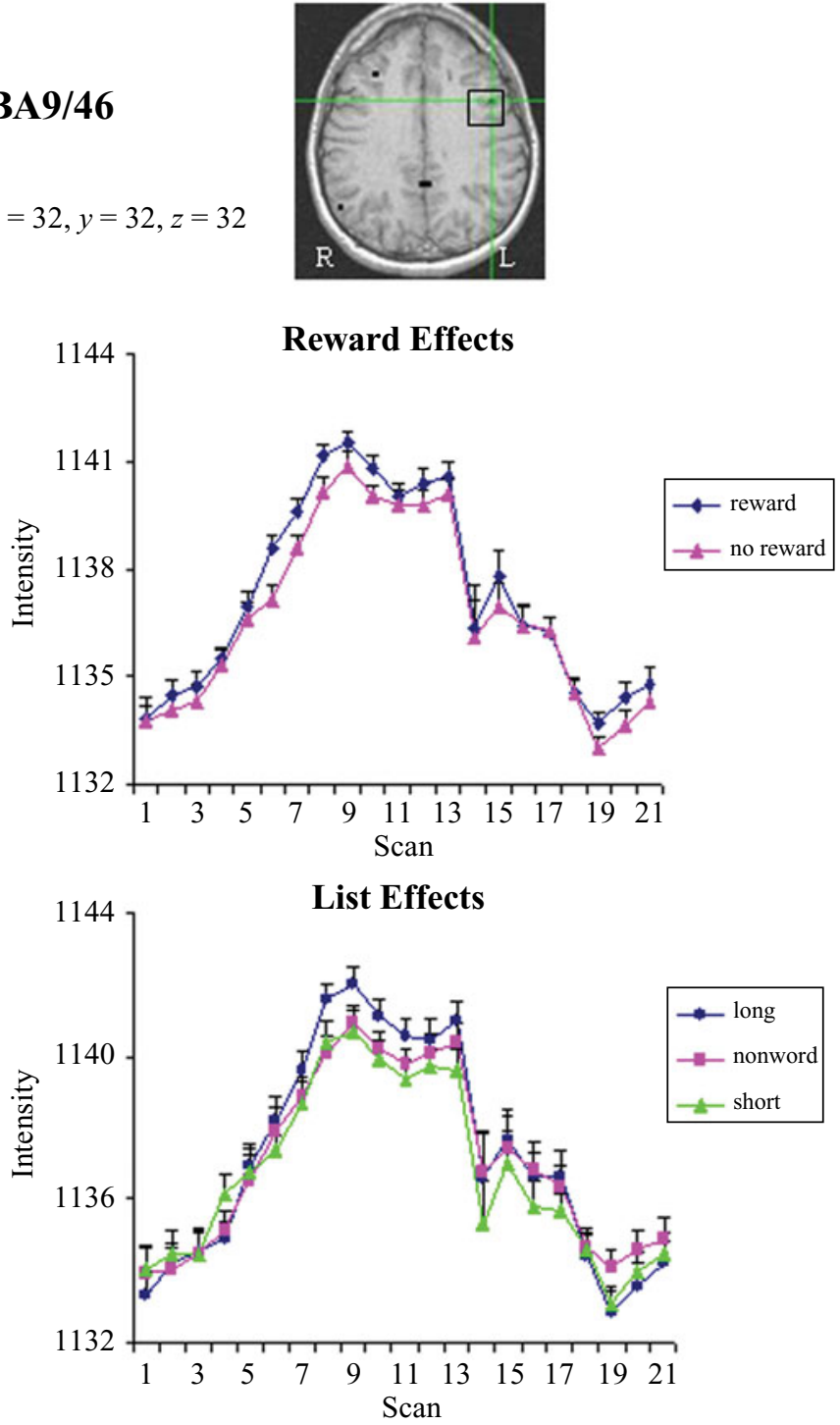

Figure 4. Reward and list type effects in DLPFC: A voxel cluster in the DLPFC (BA 9/46) showed an increase in activity during the delay in comparison with the fixation baseline epoch. As is graphically illustrated through the average time course of activity across a trial (21 scans, $63 \mathrm{sec}$ ), this cluster showed a significant effect of reward cue type (top graph) and significantly greater activation for the more difficult stimulus list conditions (bottom graph). Our analyses focused on two epochs of interest: the cue epoch (Scans 2 and 3; 3-9 sec), and the delay epoch (Scans 6-13; 15-39 sec).

$[F(1,21)=0.087, p=.77]$ or left $[F(1,21)=2.30, p=$ .14] clusters, though the conditions were ordered as predicted. Unexpectedly, we found a main effect of list type in both the right and the left clusters $[F(2,21)=3.65$, $p=.03 ; F(2,21)=5.66, p=.007]$. Since list type is not revealed until the encoding epoch of the task (Scans 3-7), these differences may reflect baseline shifts in activation caused by preceding trials.

During our delay epoch, our patterns of activation are complex. On the basis of prior neurophysiological observations of increases in neuronal firing associated with reward-related states (Hikosaka \& Watanabe, 2000; Roesch \& Olson, 2004; Schultz et al., 2000; Tremblay \& Schultz, 1999), we hypothesized that we might find significant positive activation during the delay interval. Surprisingly, however, the overall signal changes in these clusters were deactivations (delay activity less than fixation baseline). Furthermore, there was a pattern of greater deactivation for rewarded trials than for nonrewarded trials, though these differences did not reach significance for either the right $[F(1,21)=0.45, p=.51]$ or the left $[F(1,21)=3.02$, $p=.10]$ clusters. In contrast to our predictions, we found 

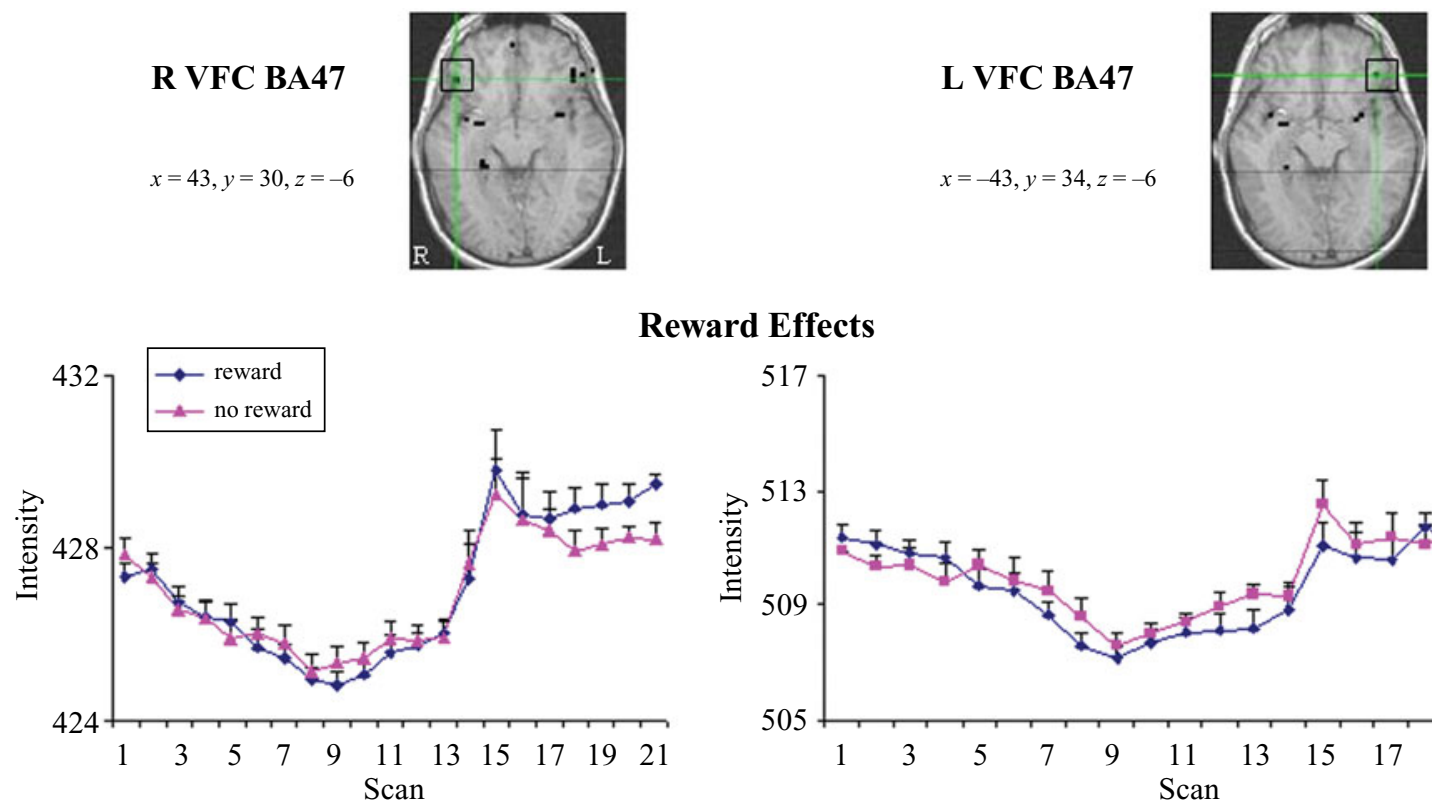

Reward Effects
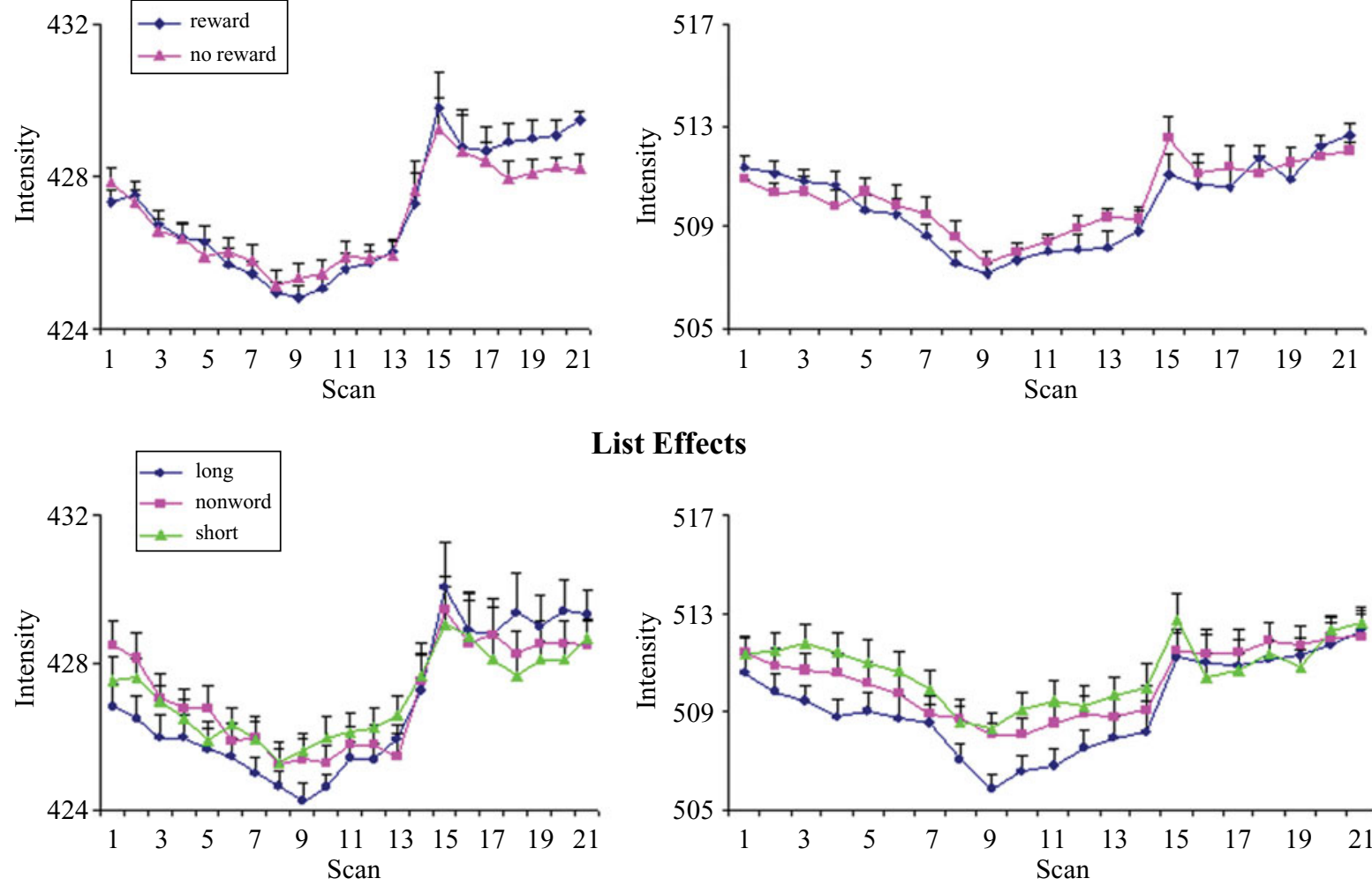

\section{List Effects}

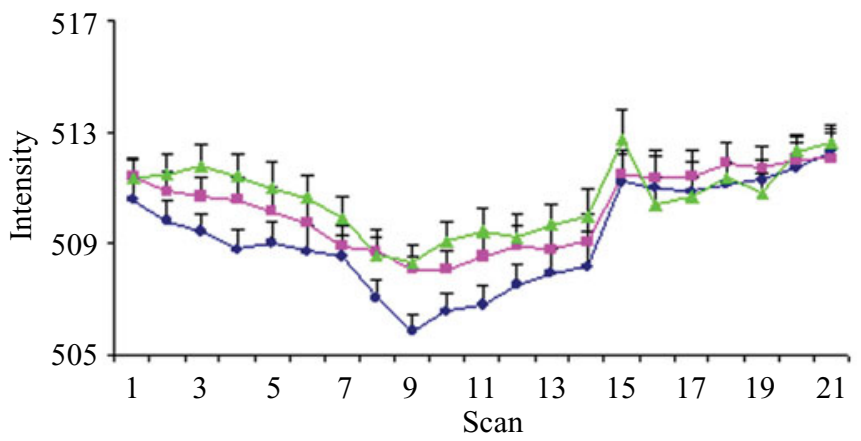

Figure 5. Reward and list type effects in the VFC: Voxel clusters located in both the right and the left VFC (BA 47) showed a decrease in activity during the delay in comparison with the fixation baseline epoch. As is graphically illustrated through the average time course of activity across a trial $(21 \mathrm{scans}, 63 \mathrm{sec})$, both clusters showed a trend toward greater deactivation for reward in comparison with no-reward trials (top graph for each cluster), and greater deactivation for the more difficult stimulus list conditions (bottom graph for each cluster). Our analyses focused on two epochs of interest: the cue epoch (Scans 2 and 3 ; 6-9 sec), and the delay epoch (Scans 6-13; 15-39 sec).

an effect of list type bilaterally $[F(2,21)=3.26, p=.05$ on the right; $F(2,21)=10.05, p<.001$ on the left]. Post hoc contrasts revealed that the deactivation in the most difficult condition (four-syllable words) was more negative than the activation in the easiest condition (twosyllable words) $[t(2,21)=-2.53, p=.020$ on the right, and $t(2,21)>-4.26, p<.001$ on the left]. There were no significant interactions between reward and list type during either the cue or the delay epoch $(p>.34)$.

The activation patterns observed in the VFC clusters were complex and unexpected. To further probe for interaction between the cue versus delay epochs and the reward condition, we conducted a post hoc voxelwise analysis across the whole brain. At each voxel, we con- ducted an ANOVA with epoch (cue vs. delay), list type, and reward condition as factors. No voxels survived the threshold ( $p<.001$, corrected) that we used in the primary omnibus contrasts of cue versus fixation and delay versus fixation. However, at a less stringent threshold ( $p<.05$, corrected) we identified four voxel clusters within PFC and VFC. Two clusters did not clearly fall within the boundaries of our a priori regions of interest; one cluster localized to BA 9/44, with a peak at $x=48$, $y=17, z=33$. The other localized to lateral BA 6 , with a peak at $x=-12, y=7, z=55$. Examination of the time courses for these regions suggests that the interactions may have emerged from baseline differences across the reward conditions. 
The two most significant clusters were in our a priori VFC region of interest, specifically in right $(x=42, y=$ $31, z=-4)$ and left $(x=-39, y=49, z=-6)$ BA 47. To further characterize the activation patterns in these clusters, two-way ANOVAs with reward condition and list type were conducted in both the cue and the delay epochs. In the cue epoch, significant effects of reward condition were observed bilaterally $[F(1,21)=7.68, p=$ .01 on the right; $F(1,21)=26.80, p<.0001$ on the left], with greater activation for the reward than for no-reward trials; the list effects did not reach significance. In the delay epoch, the reward trials were associated with a greater deactivation bilaterally, but this difference did not reach significance $[F(1,21)=3.50, p=.08$ on the right; $F(1,21)=2.93, p=.10$ on the left]. Significant list effects were found bilaterally $[F(2,21)=4.42, p=$ .02 on the right; $F(2,21)=8.50, p<.001$ on the left]. There were no significant interactions between reward and list type during either the cue or the delay epoch $(p>.35)$

\section{DISCUSSION}

In this study, our goal was to understand the relationship between frontal brain regions responsible for verbal rehearsal, executive control, and reward-related processing during a delayed serial recall task. We found that the delayed serial recall task influenced each processing domain in a manner that was consistent with the majority of our predictions.

\section{Rehearsal-Related Processing}

Turning to rehearsal-related processing, we hypothesized that frontal areas putatively engaged in verbal rehearsal would be engaged by the serial recall task, though they would be relatively insensitive to the effects of reward cues. We successfully identified clusters in the left dorsal inferior frontal cortex (BA 44/6), left ventral inferior frontal cortex (BA 45), and the supplementary motor area (BA 6) that were more active during the early maintenance epoch of the serial recall task, relative to a fixation baseline. We replicated prior findings that these regions are sensitive to stimulus manipulations that affect rehearsal difficulty across a delay interval (Chein \& Fiez, 2001). We extended this prior work to demonstrate that rehearsal-related regions were not significantly affected by reward cues, during either the initial cue epoch or an extended delay interval.

As discussed in greater detail below, the pattern of results observed in these putative rehearsal regions differs from that observed in DLPFC. Although this dissociation was predicted, the specificity of reward effects observed in this study stands in contrast to recent findings in nonhuman primates. Roesch and Olson $(2003,2004)$ found that neurons in the fontal eye fields, supplementary eye fields, premotor cortex, and supplementary motor area exhibited an increase in neuronal activity during the delay interval of a memory-guided saccade task under conditions of high motivation. The contradic- tory results may be understood by considering two different aspects of motor behavior. First, delayed recall tasks require some type of overt motor response (e.g., a delayed saccade), and incentive cues may produce increases in neuronal firing associated with motor preparation for a specific response, or a more general increase in motor arousal (Roesch \& Olson, 2003, 2004). Consistent with this interpretation, monkeys respond more quickly on high versus low incentive trials of a memoryguided saccade task (Roesch \& Olson, 2003, 2004). Second, the anticipated consumption of a reward may also change motor behavior. For instance, presentation of a conditioned cue associated with future delivery of a juice reward will initiate licking behavior in monkeys (Fiorillo, Tobler, \& Schultz, 2003). Both of these factors may have played less of a role in the present study, because participants were not under substantial time pressure to respond, nor were monetary rewards actually delivered during the course of the scanning session.

\section{Executive Control}

We hypothesized that the DLPFC would be engaged during performance of the delayed serial recall task. We successfully identified a cluster in the right, but not the left, DLPFC. This lateralization of our activity is consistent with what has been found in other studies, in which greater right as opposed to left DLPFC activation has been found using both verbal and nonverbal stimuli (Chein \& Fiez, 2001; D’Esposito et al., 1998; Rypma \& D’Esposito, 1996).

In prior research with the delayed recall task, manipulations of stimulus type were associated with weak, nonsignificant, increases in activity in the DLPFC (Chein \& Fiez, 2001). In the present study, a significant effect of list type was observed, with the degree of activation ordering inversely with task performance (i.e., the largest signal increase was observed in the most difficult condition). The magnitude of the list type effect is comparable across studies. Thus, the fact that the effect reached significance in the present study stems most likely from an increase in statistical power associated with a near doubling of the sample size (from 12 to 22 participants). Overall, the results remain consistent with a prior claim (Chein \& Fiez, 2001) that the DLPFC is more robustly affected by manipulations of memory load than by stimulus type, although this claim has yet to be tested in a within-subjects design.

An interesting new question addressed in this study is what happens when performance is manipulated by changing task incentives. Prior neurophysiological studies in nonhuman primates have suggested that task incentives can affect performance, potentially via enhanced mnemonic activity in DLPFC neurons (Kobayashi et al., 2002; Leon \& Shadlen, 1999; Roesch \& Olson, 2004). For instance, Leon and Shadlen (1999) found that monkeys demonstrated greater accuracy on rewarded trials of a memory-guided saccade task, and neurons in the DLPFC fired more strongly during the memory delay in- 
terval. In agreement with such findings, we found that recall performance was higher during rewarded than during nonrewarded trials, as was the level of activation in the DLPFC during a delay interval.

The pattern of effects observed across regions putatively engaged in verbal rehearsal and executive control provides insight into the mechanisms that support verbal working memory performance. First, the findings suggest that activity in the DLPFC does not simply reflect task performance, or task difficulty. Manipulations of memory load and stimulus type both produce decreases in recall accuracy, but increases in DLPFC activation (Braver et al., 1997; Chein \& Fiez, 2001; Rypma, Prabhakaran, Desmond, Glover, \& Gabrieli, 1999). In contrast, manipulations of incentive produce increases in both recall accuracy and DLPFC activation. Thus, activation in the DLPFC seems to correspond more closely to subjective notions of "effort." Second, the findings can be used to contrast potential mechanisms by which incentives may improve performance. One possibility is that performance is enhanced by improving verbal rehearsal. For example, it is known that working memory performance correlates with speech rate (Ellis \& Hennelly, 1980; Hulme, Thomson, Muir, \& Lawrence, 1984), and thus, potentially, subjects could increase their rate of inner speech in order to refresh decaying phonological representations more quickly and more often (White, Craft, Hale, Schatz, \& Park, 1995). A second possibility is that performance is improved by making more effective use of rehearsal. For instance, the phonological representations that may be activated by inner speech (Baddeley, 1986) might be more effectively sustained through attention or more effectively integrated with other types of information. The fact that reward effects were observed in the DLPFC, and not in rehearsal-related regions, leads us to favor the second possibility.

\section{The Role of the Ventral Frontal Cortex}

We identified clusters of activation in the right and left VFC cortex (at or near BA 47). We hypothesized that, relative to a fixation baseline, these clusters would exhibit increased activation during the cue epoch for reward versus no-reward trials. We did not observe a significant effect of reward, though the conditions did order as predicted. Furthermore, a post hoc voxelwise analysis yielded significant effects of the reward condition during the cue epoch (see Figure 6). Several factors may account for the failure to observe robust effects of reward in the VFC. First, the VFC is located near the sinuses and thus is particularly prone to susceptibility artifacts that can dramatically increase variance and decrease statistical power (Krasnow et al., 2003) (see Figure 7). Second, we collected data from only six trials of each type from each subject (18 reward and 18 no-reward trials, each divided into three different list types). This was done to minimize subject fatigue associated with extended performance of a demanding cognitive task, but as a consequence may have increased our susceptibility to Type II errors. Third, the task paradigm incorporated a relatively short cue epoch (6 sec), and, as described below, increases in activation may have been obscured by decreases in activation that occurred with the onset of a memory load. Fourth, emerging neural data suggests that the neural response to a reward cue may be relatively rapid and thus may not generate a robust hemodynamic response. Specifically, in a study by Roesch and Olson (2004), monkeys were presented with a cue that indicated the potential outcomes associated with two alternative eye movements. Unlike in many past studies of reward-related processing in the VFC, the reward cue and the mnemonic stimuli were not confounded together. Under these conditions, neurons in the VFC did not exhibit sustained activity across a delay interval and instead responded phasically to cues signaling the potential to earn a reward.

We had several hypotheses about the pattern of delay activity that might be found in the VFC. Given the findings of prior research, delay activity signaling the expectation of reward, or mnemonic processing, might be represented by positive activation. However, we found that activation during the delay decreased below baseline. A recent fMRI study by Pochon et al. (2002) found similar results and contributes to our understanding of why the VFC and DLPFC may interact to improve task performance. In Pochon et al.'s study, participants engaged in an $N$-back working memory task. In each trial of the $N$-back task, participants were presented with reward information about each trial pseudorandomly, and reward amounts increased in response to an increase in the accuracy of participants' responses.

Similar to our results, Pochon et al. (2002) found that the VFC (BA 11/47) was deactivated during rewarded trials. Furthermore, the degree of deactivation increased with increases in cognitive demand. Conversely, both rewards and increasing cognitive load were found to increase activation in the DLPFC. Pochon et al. concluded that there is a "dynamic interplay" between regions responsible for cognition and working memory and regions responsible for affective processing. Potentially, cognitively demanding trials may require the suppression of activity in the VFC in order to minimize competing emotional thoughts and emotional responses to reward (i.e., anxiety) that may interfere with task performance. As these thoughts and emotions are minimized, regions involved in executive control may function more efficiently, thereby improving task performance.

One way in which the relationship between affective and cognitive processing may be facilitated is through reciprocal neural connections between dorsal and ventral prefrontal brain regions (Cavada, Compañy, Tejedor, CruzRizzolo, \& Reinoso-Suárez, 2000; Petrides \& Pandya, 1999). Another potential mechanism would be via corticalsubcortical connections. For instance, Haber et al. (2000) have proposed a feed forward mechanism whereby mesostriatal pathways facilitate the flow of information from ventral to more dorsal striatal regions. The model is 

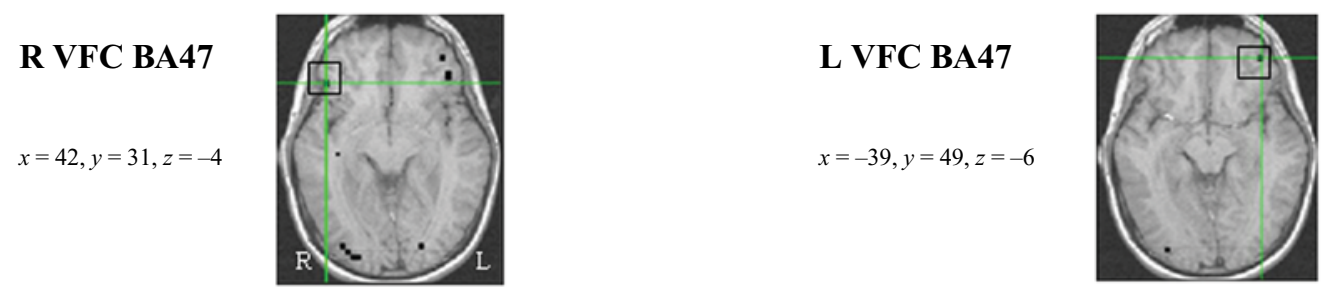

\section{Reward Effects}
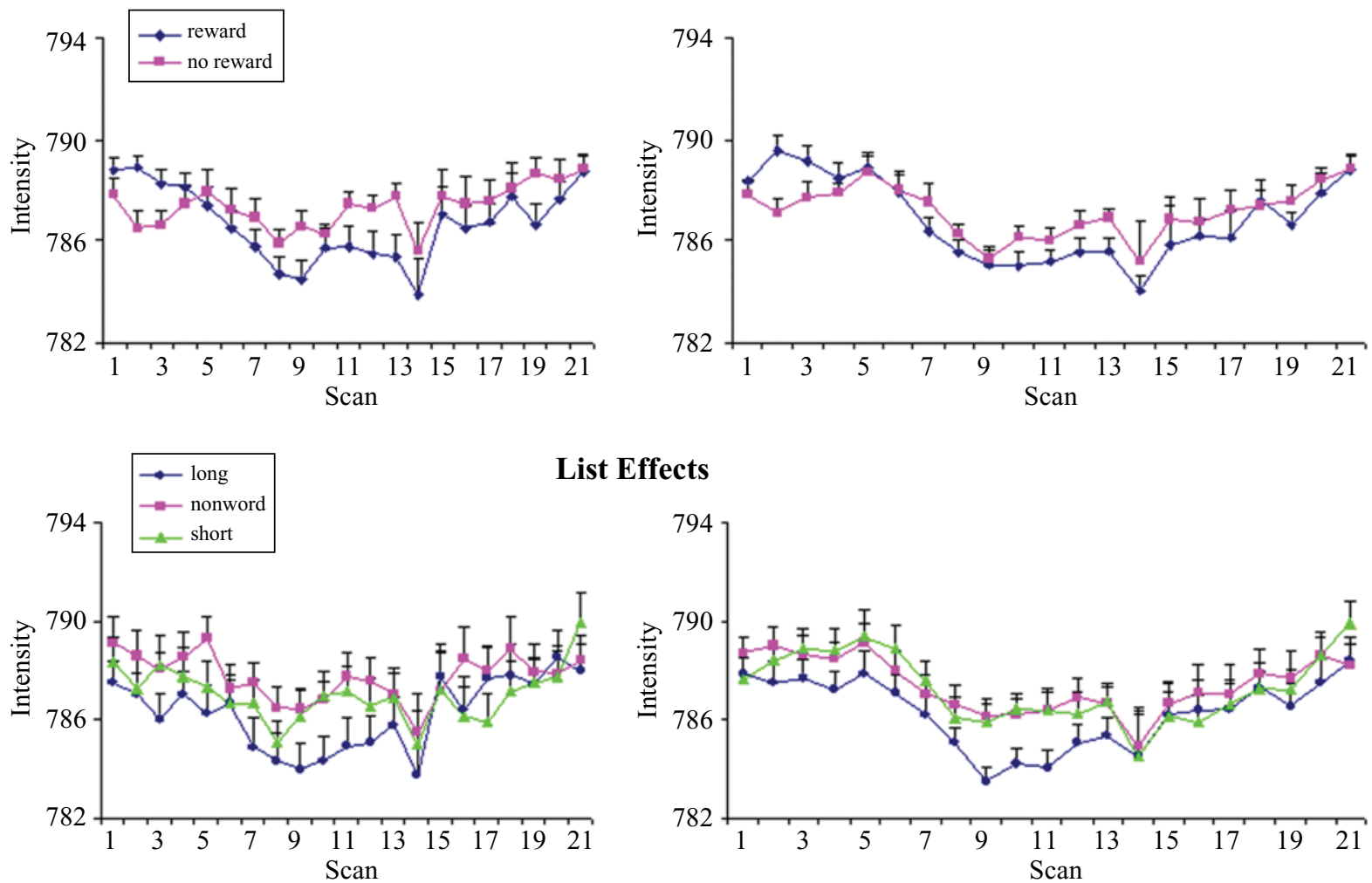

Figure 6. Post hoc voxelwise results in the VFC: A post hoc voxelwise analysis identified the left and right VFC clusters that exhibited an interaction between signal intensity in the cue (Scans 2 and 3) versus delay (Scans 8 and 9) epochs as a function of whether the trials were rewarded or unrewarded. These clusters localized to BA 47, near those identified through the primary omnibus contrast between delay and fixation (see Figure 4). As is graphically illustrated through the average time course of activity across a trial (21 scans, $63 \mathrm{sec}$ ), both clusters showed greater activation for reward as opposed to no-reward trials in the cue epoch, coupled with a trend toward a reversal of this pattern in the delay epoch (greater activation in no-reward than in reward trials) (top graphs). These clusters also exhibited significant effects of list type during the delay epoch, with the greatest deactivation observed for the more difficult stimulus lists (bottom graphs). Our analyses focused on two epochs of interest: the cue epoch (Scans 2 and 3; 3-9 sec), and the delay epoch (Scans 6-13; 15-39 sec).

based on results from anatomical tracing methods that divide the striatum into three discrete areas: ventromedial striatum, central striatum, and the dorsolateral striatum. Haber et al. propose that a "downward spiral" of communication begins in the ventral striatum (responsible for limbic processes) and influences the central striatum (responsible for cognitive processes) via overlapping connections in the midbrain. Likewise, information from the central striatum influences the dorsal striatum via shared connections in the midbrain. The ventral, central, and dorsal components of the striatal system have connections to ventral and dorsal prefrontal cortex and may enable communication between these prefrontal regions. Finally, thalamic-cortical connections may provide a mechanism for communication between prefrontal regions that respond to affective and cognitive task demands. Indeed, both the lateral and orbital prefrontal cortex have connections with the mediodorsal nucleus of the thalamus (Haber, 2003).

Research from other scientific domains further supports a relationship between affective and cognitive prefrontal processing domains. For example, clinical research has found that cognitive control, or reappraisal of phobic stimuli, involves a change in blood flow in the 

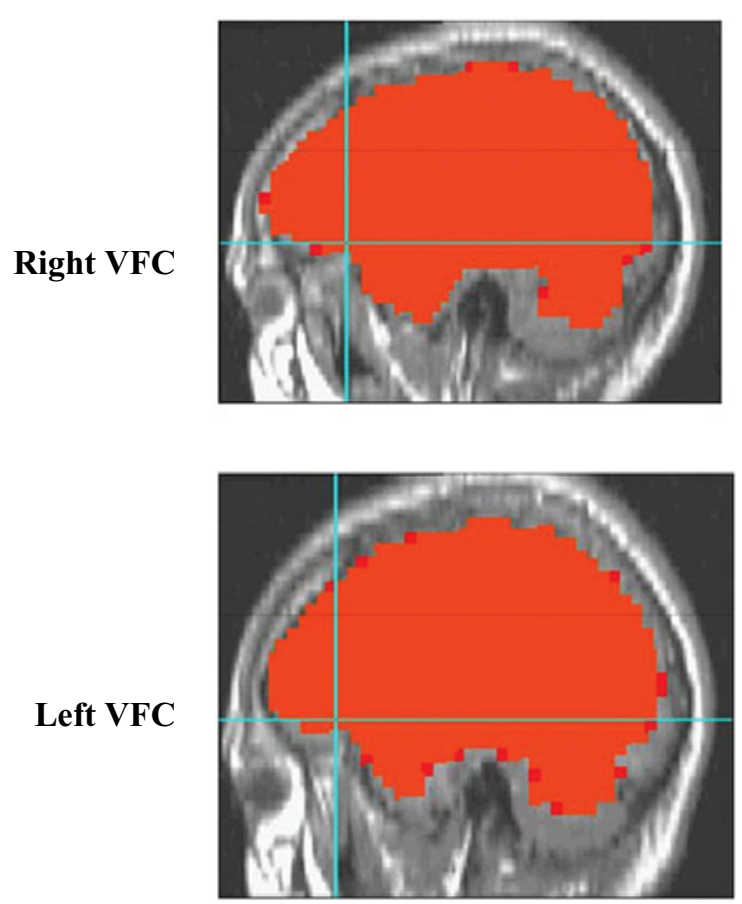

Figure 7. Map of mean raw signal intensity values; the coverage of these functional images is depicted in a voxelwise map. Voxels shown in red have signal intensity that is $50 \%$ or greater than the overall mean signal intensity value of the raw functional image averaged across subjects and conditions. The image acquisition parameters associated with this study provided nearly full-brain coverage, but as expected, data were not effectively acquired from regions associated with signal drop-out due to susceptibility artifacts (e.g., anterior portions of the VFC and the temporal lobe). The peaks of the VFC clusters identified in our primary omnibus contrast between the delay and fixation epochs are marked by the crosshairs.

DLPFC (Paquette et al., 2003). Also, research involving emotion induction in normal participants has found that cognitive reappraisal and suppression of emotional experience engages both the DLPFC and VFC (Lévesque et al., 2003; Ochsner, Bunge, Gross, \& Gabrieli, 2002). Future research could attempt to look for patterns of DLPFC-VFC interactions across different literatures. This type of analysis could provide a better understanding of how prefrontal regions respond to competing affective and cognitive demands.

In addition, future research may benefit from considering how blood flow changes exhibited in the prefrontal cortex could be part of a larger network of affective and cognitive regions. While our study focused on a priori regions of interest specifically located within frontal cortex, preliminary post hoc analyses provided some evidence that other regions - such as the parahippocampal cortex and occipital cortex - were affected by the reward manipulation. Techniques such as structural equation modeling might offer important tools for understanding the relationship between ventral prefrontal regions and other regions involved in affective processing (Northoff et al., 2004).

\section{REFERENCES}

BAdDELEY, A. D. (1986). Working memory. Oxford: Oxford University Press, Clarendon Press.

Braver, T. S., Cohen, J. D., Nystrom, L. E., Jonides, J., Smith, E. E., \& Noll, D. C. (1997). A parametric study of prefrontal cortex involvement in human working memory. Neurolmage, 5, 49-62.

Cavada, C., Compañy, T., Tejedor, J., CruZ-Rizzolo, R. J., \& ReinosoSUÁREZ, F. (2000). The anatomical connections of the macaque monkey orbitofrontal cortex: A review. Cerebral Cortex, 10, 220-242.

Chein, J. M., \& Fiez, J. A. (2001). Dissociation of verbal working memory system components using a delayed serial recall task. Cerebral Cortex, 11, 1003-1014.

Cohen, J. D., Perlstein, W. M., Braver, T. S., Nystrom, L. E., Noll, D. C., Jonides, J., \& SMith, E. E. (1997). Temporal dynamics of brain activation during a working memory task. Nature, 386, 604-608.

Cox, R. W. (1996). AFNI: Software for analysis and visualization of functional magnetic resonance neuroimages. Computational Biomedical Research, 29, 162-173.

D'Esposito, M. D., AGuirre, G. K., Zarahn, E., Ballard, D., Shin, R. K., \& LEASE, J. (1998). Functional MRI studies of spatial and nonspatial working memory. Cognitive Brain Research, 7, 1-13.

Ellis, N. C., \& Hennelly, R. A. (1980). A bilingual word-length effect: Implications for intelligence testing and the relative ease of mental calculation in Welsh and English. British Journal of Psychology, 71, 43-51.

Fiorillo, C. D., Tobler, P. N., \& Schultz, W. (2003). Discrete coding of reward probability and uncertainty by dopamine neurons. Science, 299, 1898-1902.

FrANCIS, W. N., \& KučERA, H. (1982). Frequency analysis of English usage: Lexicon and grammar. Boston: Houghton Mifflin.

Funahashi, S., Bruce, C. J., \& Goldman-Rakic, P. S. (1989). Mnemonic coding of visual space in the monkey's dorsolateral prefrontal cortex. Journal of Neurophysiology, 61, 331-349.

Haber, S. N. (2003). The primate basal ganglia: Parallel and integrative networks. Journal of Chemical Neuroanatomy, 26, 317-330.

Haber, S. N., FudGe, J. L., \& McFarLAND, N. R. (2000). Striatonigrostriatal pathways in primates form an ascending spiral from the shell to the dorsolateral striatum. Journal of Neuroscience, 20, 2369-2382.

Hikosaka, K., \& Watanabe, M. (2000). Delay activity of orbital and lateral prefrontal neurons of the monkey varying with different rewards. Cerebral Cortex, 10, 263-271.

Hulme, C., Thomson, N., Muir, C., \& Lawrence, A. (1984). Speech rate and the development of short-term memory span. Journal of Experimental Child Psychology, 38, 241-253.

Johnston, D. (2000). Cool Edit 2000. Phoenix, AZ: Syntrillium Software Corporation.

Kobayashi, S., Lauwereyns, J., Koizumi, M., SaKagami, M., \& Hikosaka, O. (2002). Influence of reward expectation on visuospatial processing in macaque lateral prefrontal cortex. Journal of Neurophysiology, 87, 1488-1498.

Krasnow, B., Tamm, L., Greicius, M. D., Yang, T. T., Glover, G. H., Reiss, A. L., \& MEnON, V. (2003). Comparison of fMRI activation at 3 and $1.5 \mathrm{~T}$ during perceptual, cognitive, and affective processing. NeuroImage, 18, 813-826.

LEON, M. I., \& SHADLEN, M. N. (1999). Effect of expected reward magnitude on the response of neurons in the dorsolateral prefrontal cortex of the macaque. Neuron, 24, 415-425.

Lévesque, J., Eugene, F., JoAnette, Y., Paquette, V., Mensour, B., Beaudoin, G., Leroux, J. M., Bourgouin, P., \& Beauregard, M. (2003). Neural circuitry underlying voluntary suppression of sadness. Biological Psychiatry, 53, 502-510.

Northoff, G., Kotter, R., Baumgart, F., Danos, P., Boeker, H., Kaulisch, T., Schlagenhauf, F., Walter, H., Heinzel, A., Witzel, T., \& Bogerts, B. (2004). Orbitofrontal cortical dysfunction in akinetic catatonia: A functional magnetic resonance imaging study during negative emotional stimulation. Schizophrenia Bulletin, 30, 405-427.

OCHSNer, K. N., Bunge, S. A., Gross, J. J., \& Gabrieli, J. D. E. (2002). Rethinking feelings: An fMRI study of cognitive regulation of emotion. Journal of Cognitive Neuroscience, 14, 1215-1229. 
Otтo, T., \& Eichenbaum, H. (1992). Complementary roles of the orbital prefrontal cortex and the perirhinal-entorhinal cortices in an odor-guided delayed-nonmatching-to-sample task. Behavioral Neuroscience, 106, 762-775.

Paquette, V., LéVesque, J., Mensour, B., Leroux, J.-M., Beaudoin, G., Bourgouin, P., \& Beauregard, M. (2003). "Change the mind and you change the brain": Effects of cognitive-behavioral therapy on the neural correlates of spider phobia. NeuroImage, 18, 401-409.

Perlstein, W. M., Elbert, T., \& Stenger, A. V. (2002). Dissociation in human prefrontal cortex of affective influences on working memoryrelated activity. Proceedings of the National Academy of Sciences, 99, 1736-1741.

PeTRIDES, M., \& PANDYA, D. N. (1999). Dorsolateral prefrontal cortex: Comparative cytoarchitectonic analysis in the human and the macaque brain and corticocortical connection patterns. European Journal of Neuroscience, 11, 1011-1036.

Pochon, J. B., Levy, R., Fossati, P., Lehericy, S., Poline, J. B., PilLON, B., Le Bihan, D., \& Dubois, B. (2002). The neural system that bridges reward and cognition in humans: An fMRI study. Proceedings of the National Academy of Sciences, 99, 5669-5674.

Roesch, M. R., \& Olson, C. R. (2003). Impact of expected reward on neuronal activity in prefrontal cortex, frontal and supplementary eye fields and premotor cortex. Journal of Neurophysiology, 90, 17661789.

Roesch, M. R., \& Olson, C. R. (2004, April). Neuronal activity related to reward value and motivation in primate frontal cortex. Science, 304, 307-310.

RoLls, E. T. (1999). The brain and emotion. Oxford: Oxford University Press.

Rypma, B., \& D'Esposito, M. (1996). The roles of prefrontal brain regions in components of working memory: Effects of memory load and individual differences. Proceedings of the National Academy of Sciences, 96, 6558-6563.

Rypma, B., Prabhakaran, V., Desmond, J. E., Glover, G. H., \& GABRIELI, J. D. E. (1999). Load-dependent roles of frontal brain re- gions in the maintenance of working memory. NeuroImage, 9, 216226.

Schoenbaum, G., \& Setlow, B. (2001). Integrating orbitofrontal cortex into prefrontal theory: Common processing themes across species and subdivisions. Learning \& Memory, 8, 134-147.

Schultz, W., Tremblay, L., \& Hollerman, J. R. (2000). Reward processing in primate orbitofrontal cortex and basal ganglia. Cerebral Cortex, 10, 272-283.

Sharp, D. J., Scott, S. K., \& Wise, R. J. S. (2004). Monitoring and the controlled processing of meaning: Distinct prefrontal systems. Cerebral Cortex, 14, 1-10.

Tremblay, L., \& Schultz, W. (1999). Relative reward preference in primate orbitofrontal cortex. Nature, 398, 704-708.

Tremblay, L., \& Schultz, W. (2000). Reward-related neuronal activity during go-nogo task performance in primate orbitofrontal cortex. Journal of Neurophysiology, 83, 1864-1876.

WARD, D. B. (2000). Simultaneous inference for MRI data [On line]. Available at http://afni.nimh.nih.gov/pub/dist/doc/manuals/Alpha Sim.pdf.

WATANABE, M. (1996). Reward expectancy in primate prefrontal neurons. Nature, 382, 629-632.

Watanabe, M., HikosaKa, K., Sakagami, M., \& Shirakawa, S.-I. (2002). Coding and monitoring of motivational context in the primate prefrontal cortex. Journal of Neuroscience, 22, 2391-2400.

White, D. A., Craft, S., Hale, S., Schatz, J., \& PARK, T. S. (1995). Working memory following improvements in articulation rate in children with cerebral palsy. Journal of the International Neuropsychological Society, 1, 49-55.

Woods, R. P., Mazziotta, J. C., \& Cherry, S. R. (1993). MRI-PET registration with automated algorithm. Journal of Computer Assisted Tomography, 17, 536-546.

(Manuscript received August 10, 2004; revision accepted for publication November 11, 2004.) 